OPEN ACCESS

Edited by:

Jean-Christophe Avarre, Institut de Recherche pour le Développement (IRD), France

Reviewed by:

Hetron Mweemba Munang'andu, Norwegian University of Life Sciences,

Norway

Laura S. Epp

Universität Konstanz, Germany

*Correspondence:

Martin S. Llewellyn

martin.llewellyn@glasgow.ac.uk

${ }^{\dagger}$ These authors have contributed equally to this work

Specialty section:

This article was submitted to

Aquatic Microbiology,

a section of the journal

Frontiers in Microbiology

Received: 01 February 2018 Accepted: 20 November 2018 Published: 07 December 2018

Citation:

Peters L, Spatharis S, Dario MA,

Dwyer T, Roca IJT, Kintner A, Kanstad-Hanssen $\varnothing$, Llewellyn MS and Praebel K (2018) Environmental DNA: A New Low-Cost Monitoring

Tool for Pathogens in Salmonid Aquaculture. Front. Microbiol. 9:3009. doi: 10.3389/fmicb.2018.03009

\section{Environmental DNA: A New Low-Cost Monitoring Tool for Pathogens in Salmonid Aquaculture}

\author{
Lucy Peters' ${ }^{1,2 \dagger}$, Sofie Spatharis $2,3+$, Maria Augusta Dario ${ }^{4}$, Toni Dwyer ${ }^{2}$, Inaki J. T. Roca ${ }^{5}$, \\ Anna Kintner ${ }^{2}$, Øyvind Kanstad-Hanssen ${ }^{6}$, Martin S. Llewellyn ${ }^{2 * t}$ and Kim Praebel'5t \\ ${ }^{1}$ Institute of Evolutionary Biology, University of Edinburgh, Edinburgh, United Kingdom, ${ }^{2}$ Institute of Biodiversity, Animal \\ Health and Comparative Medicine, University of Glasgow, Glasgow, United Kingdom, ${ }^{3}$ School of Life Sciences, University \\ of Glasgow, Glasgow, United Kingdom, ${ }^{4}$ Laboratório de Biologia de Tripanosomatídeos, Instituto Oswaldo Cruz \\ (IOC/Fiocruz), Rio de Janeiro, Brazil, ${ }^{5}$ Norwegian College of Fishery Science, University of Tromsø, Tromsø, Norway, \\ ${ }^{6}$ Ferskvannsbiologen, Ltd., Lødingen, Norway
}

Environmental DNA (eDNA) metabarcoding is a relatively new monitoring tool featuring in an increasing number of applications such as the facilitation of the accurate and cost effective detection of species in environmental samples. eDNA monitoring is likely to have a major impact on the ability of salmonid aquaculture industry producers and their regulators to detect the presence and abundance of pathogens and other biological threats in the surrounding environment. However, for eDNA metabarcoding to develop into a useful bio-monitoring tool it is necessary to (a) validate that sequence datasets derived from amplification of metabarcoding markers reflect the true species' identity, (b) test the sensitivity under different abundance levels and environmental noise and (c) establish a low-cost sequencing method to enable the bulk processing of field samples. In this study, we employed an elaborate experimental design whereby different combinations of five biological agents were crossed at three abundance levels and exposed to sterile pre-filtered and unfiltered seawater, prior to coarse filtering and then eDNA ultrafiltration of the resultant material. We then benchmarked the low-cost, scalable, Ion Torrent sequencing method against the current gold-standard Illumina platform for eDNA surveys in aquaculture. Based on amplicon-seq of the 18S SSU rDNA v9 region, we were able to identify two parasites (Lepeophtheirus salmonis and Paramoeba perurans) to species level, whereas the microalgae species Prymnesium parvum, Pseudo-nitzschia seriata, and $P$. delicatissima could be assigned correctly only to the genus level. Illumina and lon Torrent provided near identical results in terms of community composition in our samples, whereas lon Torrent was more sensitive in detecting species richness when the medium was unfiltered seawater. Both methods were able to reflect the difference in relative abundance between treatments in 4 out of 5 species when samples were exposed to the unfiltered seawater, despite the significant 
amount of background noise from both bacteria and eukaryotes. Our findings indicate that eDNA metabarcoding offers significant potential in the monitoring of species harmful to aquaculture and for this purpose, the low-cost lon Torrent sequencing is as accurate as Illumina in determining differences in their relative abundance between samples.

\section{Keywords: Pseudo-nitzschia seriata, Prymnesium parvum, Lepeophtheirus salmonis, Paramoeba perurans,} environmental DNA (eDNA), aquaculture

\section{INTRODUCTION}

The salmonid aquaculture industry is undergoing explosive growth globally. However, the industry is beset by parasitic disease and is often the subject to mass mortalities of farmed fish due to toxin-producing Harmful Algal Blooms (HABs) (Smayda, 2006; Hinder et al., 2011). Economic losses associated with certain agents, as for example sea lice, accounts for up to $£ 470$ M/year for major producers like Norway (Liu and Bjelland, 2014). The presence and abundance thresholds of these potentially damaging organisms in the environment and around aquaculture sites must be closely monitored. Traditional microscopy methods for algal and copepod larval species identification are timeconsuming, demand expertise and are not always accurate when abundances are low or when cryptic species are involved. Similarly, parasite counts on the fish themselves are both time consuming and impose significant handling stress.

Environmental DNA (eDNA) analysis is an emerging molecular approach for species identification from samples containing cellular DNA and extracellular DNA sloughed off all living organisms (Bohmann et al., 2014). eDNA analysis has been successfully employed to detect and monitor eukaryotic micro- and macrobial communities and populations (Ficetola et al., 2008; Thomsen et al., 2012; Zamor et al., 2012) and is a useful tool for early monitoring systems as it allows for more accurate and standardized detection of species that are cryptic, inaccessible (Thomsen et al., 2012) and of low abundance (Ficetola et al., 2008). There have been several studies that have validated eDNA as a monitoring tool (Young et al., 2008; Olson et al., 2012; Zamor et al., 2012; Mahon et al., 2013; Wood et al., 2013) with some recent advances toward its application for pathogen detection in freshwater aquaculture (Gomes et al., 2017). However, before it can be considered as a systematic bio-monitoring tool it is necessary to find a cost-effective analytical approach to allow rapid processing of environmental samples on a day-to-day basis. Furthermore, validation is required to establish the relationship between eDNA genotype data and biological abundance (e.g., Nathan et al., 2014).

Next generation sequencing (NGS) methods are increasingly being used to identify aquatic organisms from eDNA samples (Eiler et al., 2013; Yu et al., 2015). Metabarcoding - the use of universal primers to amplify DNA from many different organisms within one sample - is the technique most frequently deployed in the context of NGS (Taberlet et al., 2012). Multiplex of samples via the inclusion of molecular identifier tags allows for parallel processing of multiple samples both during the sequencing run as well as during the downstream bioinformatic analysis using a technique termed amplicon-seq (Wood et al., 2013). Two commonly used NGS methods are Ion Torrent and Illumina MiSeq - the efficiency of which in detecting organisms from community samples has been compared in multiple studies mostly focused on bacterial diversity (e.g., Salipante et al., 2014; Clooney et al., 2016; Lahens et al., 2017). Several hundred samples can be multiplexed on a single Illumina MiSeq run, but the cost per run is relatively high. The Ion Torrent platform offers scalable sequencing runs, which provides more flexibility for running low numbers of samples (20-40 per chip) on a regular basis. Most of comparative studies suggest that Illumina and Ion Torrent have similar capacities to detect changes in biology from a treatment/control experiment, however, Ion Torrent seems to be more prone to error due to organism-related biases that lead to underrepresentation of certain species (Salipante et al., 2014). This error has been attributed to the premature truncation of sequences during semiconductor sequencing (Salipante et al., 2014).

Although detection of pathogens would be an important step forward for the aquaculture industry, it is also important to be able to assess whether the abundances of target pathogenic organisms have exceeded established thresholds regarding food safety or water quality ${ }^{1}$. Species quantification remains a holy grail for eDNA studies and several authors report progress toward this goal in aquatic organisms (Nathan et al., 2014), with more recent studies incorporating models of DNA shedding and degradation (Sassoubre et al., 2016). Absolute individuallevel quantification is complicated in comparisons of metazoans and unicellular species where biomass, instead of count data, is likely to show better correlation with DNA quantity (Sassoubre et al., 2016). More recently, data processing techniques such as normalization of read data have provided a better representation of absolute abundances across experimental treatments (Weber and Pawlowski, 2013).

In this study, our aim was to establish whether eukaryotic aquaculture pathogens and harmful algae can be reliably detected and identified using a universal metabarcoding approach. Specifically, our objectives were to (1) test whether multiple pathogens can be detected simultaneously against a background of biological "noise" from the zooplankton, (2) establish whether artificially generated differences in relative proportion/abundance of the pathogens between samples can be detected, and (3) explore the power of different sequencing methodologies to generate the data. To achieve these aims we used an elaborate experimental design involving

\footnotetext{
${ }^{1}$ http://www.gov.scot/Topics/marine/Fish-Shellfish/bill
} 
cross treatments of the target organisms at three abundance levels, exposed to background noise influence (i.e., unfiltered sea-water) versus sterile pre-filtered sea-water. We then benchmarked Illumina MiSeq and Ion Torrent techniques to deep sequence the ribosomal 18rRNA marker gene of these samples.

\section{MATERIALS AND METHODS}

\section{Choice of Pathogen}

Five major pathogens and risk agents were selected: Lepeophtheirus salmonis and Paramoeba perurans as well as the algal risk agents Prymnesium parvum (identified morphologically in University of the Aegean), Pseudo-nitzschia seriata (identified by N. Lundholm, personal communication), and P. delicatissima (CCAP culture). L. salmonis is one of the most important and widespread, affecting farmed Atlantic salmon in Norway, Ireland, and the United Kingdom (Torrissen et al., 2013; Ellis et al., 2016). P. perurans is the causative agent of amoebic gill disease, which is a major source of commercial loss for aquaculture in Tasmania, but also affects industries in both North and South America as well as in Europe (Young et al., 2008). The haptophyte $P$. parvum, produces compounds known as prymnesins causing severe toxic effects by affecting plasma membrane integrity of gills (Manning and La Claire, 2010). $P$. parvum blooms resulted in extensive mortality of farmed salmon in Scotland and Norway in the past (Smayda, 2006). Pseudo-nitzschia produce the neurotoxin Domoic acid (DA), that causes Amnesic Shellfish Poisoning (ASP) symptoms in humans (Hinder et al., 2011) when it bioaccumulates in the tissue of bivalves.

\section{Incubations and Filtering}

We incubated cultures of the different pathogens in four different groups: Group 1 containing L. salmonis, Group 2 containing $P$. perurans, Group 3 containing the combination of the three algae ( $P$. parvum, $P$. seriata, and $P$. delicatissima) and group 4 containing a mix of all the species. Each of the four groups was further divided into three treatment regimens of the following abundances: two, six and eighteen, in the case of the salmon louse referring to number of individuals (females without egg strings), and to cell counts for the amoeba and algal species. The densities of the cultures of amoeba and algal species were determined by microscopy and dilution series in a Neubauer chamber. As a negative control, a blank baseline treatment was set up for each of the two different days of the incubations. All incubations consisted of $2.0 \mathrm{~L}$ of two different media; sterile filtered $(0.22 \mu \mathrm{m})$ seawater and unfiltered seawater. In this way, the strength of the relationship between known abundances and sequence read numbers could be tested both for the baseline level of PCR amplification only, as simulated by the filtered medium, and in the context of the natural environment, replicated by the unfiltered medium. Both the unfiltered and filtered seawater were obtained from internal pipes of the marine laboratory of the Norwegian College of Fishery Science, UiT the Arctic University of Norway. Our

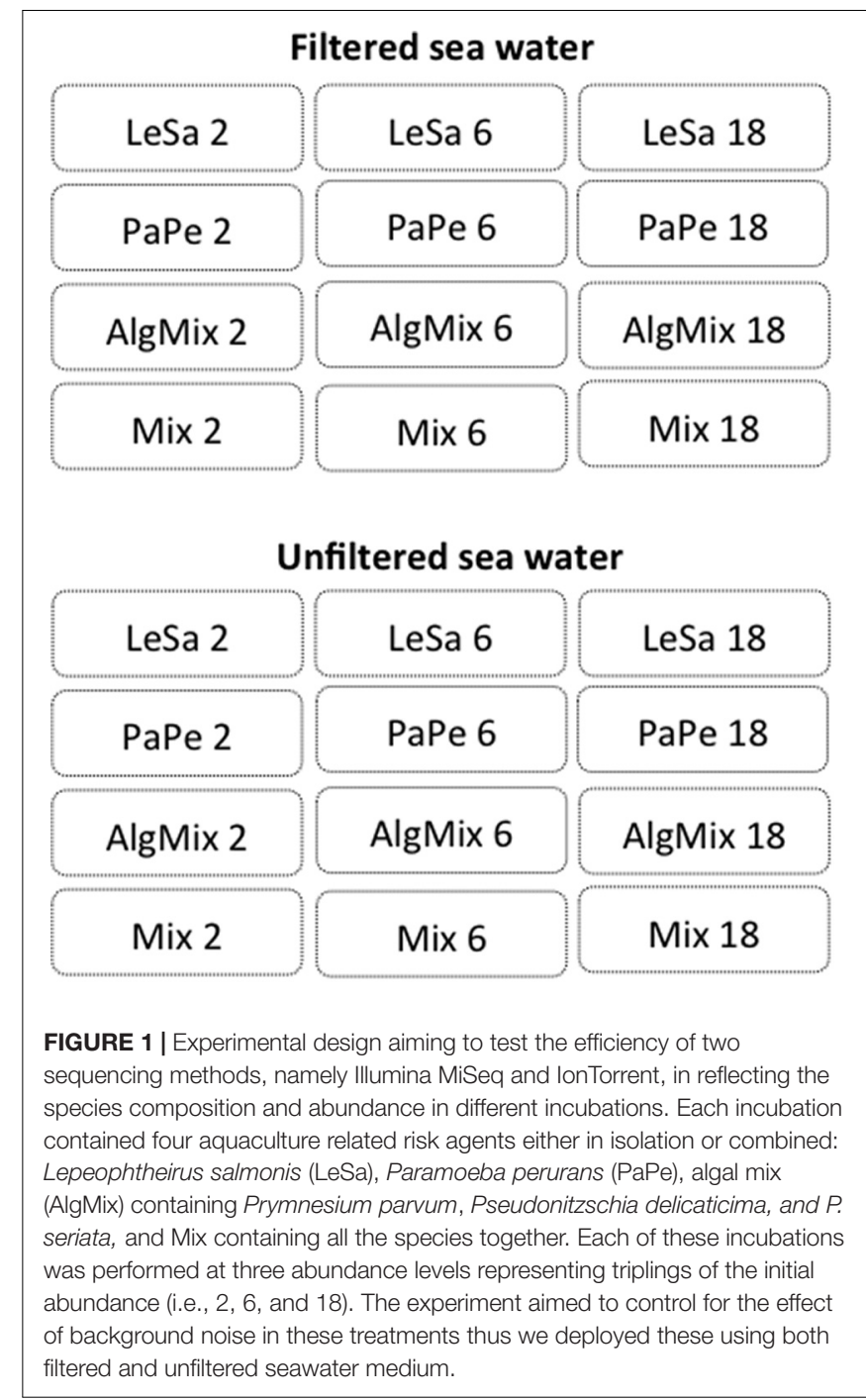

experimental design generated 72 samples ( 2 medium types: filtered/unfiltered $\times 12$ treatments $\times 3$ replicates) (see Figure 1 for experimental design). For each medium type, we also used 2 blanks replicated thrice ( 12 samples in total), resulting in a total of 84 samples. All incubations were performed at $3.8^{\circ} \mathrm{C}$ for $24 \mathrm{~h}$, before $0.5 \mathrm{~L}$ were filtered to collect material for DNA extraction and sequencing through a $0.22 \mu \mathrm{m}$ sterivex filter unit (EMD Millipore, Darmstadt, Germany). Samples for all incubations were obtained in triplicate.

\section{DNA Extraction, PCR, and Sequencing}

Total DNA was extracted using the DNeasy Blood and Tissue kit (Qiagen, Hilden, Germany) following the manufacturer's instructions with the minor adjustments. Briefly, $500 \mu \mathrm{l}$ instead of $200 \mu \mathrm{l}$ digestion buffer ATL/proteinase K was added directly to each Sterivex filter and incubated over night with continues rotation. The buffer, containing the eDNA, was then spun out of the filters into a $2 \mathrm{ml}$ Eppendorf tube at $1700 \times g$ for $3 \mathrm{~min}$. Each sample, was then added an equal volume as the eluate of the lysis buffer AL and 100\% ethanol and vortexed. The mixture 
was transferred to a spin column and the manufactures protocol followed until the elution step where $75 \mu \mathrm{lEB}$ was used instead of $200 \mu \mathrm{l}$. All handling of samples, from sampling water to extraction of eDNA and handling of extracts, was performed under strict clean conditions at designated clean labs for eDNA work at the Norwegian College of Fishery Science.

Sequencing libraries were generated using two rounds of PCR amplification. For the initial round of amplification, each PCR was conducted in a $25 \mu \mathrm{l}$ reaction volume containing 10ng of template DNA, $0.5 \mu \mathrm{M}$ of each primer and 12.5 units of Q5 Hot Start High-Fidelity 2X Master Mix (New England Biolabs: Ipswich, MA, United States). The following primers were used in the PCR reactions to amplify the $18 \mathrm{~S}$ V9 region (Hadziavdic et al., 2014): forward primer 1391_F (5'-GTACACACCGCCCGTC-3') and reverse primer 1560_R (5'-TGATCCTTCTGCAGGTTCACCTAC- $\left.3^{\prime}\right)$ with the following conditions: one initial cycle of $15 \mathrm{~min}$ at $95^{\circ} \mathrm{C}$; 35 cycles of $45 \mathrm{~s}$ at $95^{\circ} \mathrm{C}, 45 \mathrm{~s}$ at $58^{\circ} \mathrm{C}$, and $60 \mathrm{~s}$ at $72^{\circ} \mathrm{C}$; and one final cycle of $10 \mathrm{~min}$ at $72^{\circ} \mathrm{C}$. Multiplex identifier tags were added to the first round PCR products by a second, five cycle, PCR reaction $\left(45 \mathrm{~s}\right.$ at $95^{\circ} \mathrm{C}, 45 \mathrm{~s}$ at $58^{\circ} \mathrm{C}$, and $60 \mathrm{~s}$ at $\left.72^{\circ} \mathrm{C}\right)$. The $\mathrm{PCR}$ profile of the fragment amplification for Ion Torrent sequencing only differed from this protocol in the duration of the different temperature phases within the PCR cycle, which were $30 \mathrm{~s}$ for each of the three phases. The PCR products were then run on a $2 \%$ agarose gel for quality control using SYBR safe (Thermo Fisher Scientific, Waltham, MA, United States) as an in-gel stain at $\mathrm{x} 1$ concentration and DNA bands were then visualized for inspection under UV light.

Illumina MiSeq paired-end sequencing of the $18 \mathrm{~S}$ V9 region was carried out at the Glasgow Polyomics lab (Glasgow, Scotland, United Kingdom) using the MiSeq reagent kit (600 cycle) (Illumina, San Diego, CA, United States) and $2 \times 300 \mathrm{bp}$ sequencing. Ion Torrent sequencing of the amplicons was carried out in the Systems Biology Centre of the University of Plymouth (Plymouth, England, United Kingdom) on Ion 318v2 Chips, using the Ion PGM Sequencing 200 Kit v2 (Life Technologies Ltd., Carlsbad, CA, United States) for sequencing of up to $200 \mathrm{bp}$. For both sequencing procedures adapter sequences were trimmed automatically before the sequence data was exported as FASTQ files.

\section{Sequence Processing, OTU Clustering, and Taxonomic Assignment}

Raw reads from the Illumina MiSeq and the Ion Torrent run were processed in the same manner using identical parameters, apart from some differences in pre-processing of the paired-end reads of the Illumina run. All raw reads, single for the Ion Torrent and both paired ends for the MiSeq run, were trimmed with Sickle version 1.33 (Joshi and Fass, 2011) using a quality threshold of 20 and a minimum length of 100 base pairs. For the paired ends, a file with singletons was created at this step, which was excluded from further analysis. The reads were further trimmed using FASTX-Toolkit version 0.0.14 (Hannon Lab) to a maximum length of 200 base pairs. All sequences were then aligned to a reference consisting of representative $18 \mathrm{~S}$ sequences of all target organisms or a closely related species (GenBank accession numbers: AF208263.1 (Lepeophtheirus salmonis); KT989881.1 (Paramoeba perurans); KJ756812.1 (Prymnesium parvum); JF308618.1 (Pseudo-nitzschia seriata); EU478793.1 (Pseudo-nitzschia delicatissima) in an attempt to filter out non$18 \mathrm{~S}$ reads. The alignment was carried out in bowtie 2 version 2.2.6 (Langmead and Salzberg, 2012) using the low stringency local alignment option.

The matched reads then underwent further quality checking using PRINSEQ-lite version 0.20.4 (Schmieder and Edwards, 2011) to identify any formatting errors and remove read headers as well as convert the sequences into FASTA format to facilitate downstream processing. The paired-end MiSeq reads were further processed by merging the mate pairs into one sequence using Velvet version 1.2.09 (Zerbino and Birney, 2008) after reversing and complementing reads two. Merged MiSeq and single Ion Torrent reads were further processed using USEARCH version 8.1.1861 (Edgar, 2010): reads were scanned for unique sequences, sorted and finally clustered into operational taxonomic units (OTUs) using the UPARSE algorithm and a 97\% identity threshold.

A table listing the OTUs and read frequency for every sample was constructed for each of the two sequencing methods. Taxonomic identity of the OTUs was assigned in Qiime version 1.9.1 (Caporaso et al., 2010) using the closed reference approach with the SILVA database release 128 (Quast et al., 2013) as a reference, which is the most comprehensive database for eukaryotic $18 \mathrm{~S}$ sequences, and the BLAST algorithm for assignment. Multiple identical assignments for different OTUs were pooled together using Primer6 version 6.1.4 (Clarke and Gorley, 2006).

Sequence reads generated in this study have been submitted to the NCBI short-reads archive (SRA), accession number PRJNA505454.

\section{Sequencing Efficiency of MiSeq Versus Ion Torrent}

Sequencing success of MiSeq sequencing compared to Ion Torrent sequencing was quantified by the percentage of raw reads retained after quality filtering as well as by total numbers of unique OTUs identified. Overall sequencing output and sample composition for both sequencing methods were further explored by determining absolute and relative read abundances using the phyloseq package version 1.18.0 (McMurdie and Holmes, 2013) in the statistical analysis software $\mathrm{R}$ version 3.3.0 (R Core Team, 2016). The effect of treatment, sequencing method and medium type on the number of OTUs and evenness index J was tested using General Linear Models of the form:

$$
\begin{gathered}
\text { OTU richness }=\text { Method }_{\mathrm{i}}+\text { Medium }_{\mathrm{j}}+\text { Treatment }_{\mathrm{k}} \text {. } \\
\text { Evenness richness }=\text { Method }_{\mathrm{i}}+\text { Medium }_{\mathrm{j}}+\text { Treatment }_{\mathrm{k}} .
\end{gathered}
$$

Where: method is a categorical variable with two levels ( $\mathrm{i}$ = Illumina MiSeq, Ion Torrent), Medium is a categorical variable with two levels $(j=$ filtered, unfiltered), Treatment is a 


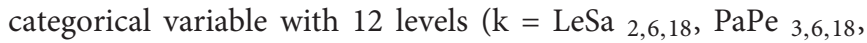
AlgMix 3,6,18, Mix 3,6,18).

\section{Sensitivity of MiSeq Versus Ion Torrent to Reflect Sample Composition}

Potential differences between the two sequencing methods in reflecting OTU composition in the samples was explored using multivariate statistics. For this analysis the OTU data were first normalized to account for inter-sample variation in sequencing depth. Data normalization was conducted in the $\mathrm{R}$ package Deseq2 version 1.14.0 (Love et al., 2014) with which instead of rarefying, a variance stabilizing transformation was applied to the read numbers to convert the counts so that they are of homoscedastic distribution, after the recommendations of McMurdie and Holmes (2014). The normalized read samples were then analyzed within each method for pairwise similarity using the Bray-Curtis similarity index. The pairwise similarities between all samples were then visualized using Multidimensional Scaling Ordination (MDS).

\section{Sensitivity of MiSeq Versus Ion Torrent to Reflect Differences in Relative Abundances Between Treatments}

The two sequencing methods of MiSeq and Ion Torrent were compared in their efficiency in reflecting the relative abundances of species within each medium type, i.e., filtered and unfiltered medium using a General Linear model of the form:

$$
\text { Species/OTU reads }=\text { Treatment }_{\mathrm{k}} \text {. }
$$

Where $\mathrm{k}=2,6$, and 18 abundance levels. The first order interaction between treatment and method and treatment and medium was also tested in order to check whether the effect of treatment depends on which method or which medium is being used. The relative (normalized) reads were used for this analysis and for the unfiltered samples we subtracted background concentrations of our target species that were found in the blanks from the abundances found in the treatments. No blank contained more than six reads from any one target species.

\section{RESULTS}

\section{Sequencing Efficiency and Taxonomic Assignment of MiSeq Versus Ion Torrent}

The Illumina platform successfully sequenced 68 out of the 84 samples whilst Ion Torrent returned a slightly lower number (62 out of the 84 samples). The two sequencing approaches showed differences in their raw read numbers and overall sequence read quality. The Illumina MiSeq data consisted of fewer raw reads $(6,115,810$ paired reads) across all samples compared with the Ion Torrent output (9,350,400 single reads). After quality filtering of the raw reads, $93.7 \%$ of the MiSeq sequence pairs were kept compared to just $68.3 \%$ of the Ion Torrent raw reads. As a result the total number of reads that passed QC were similar for both sequencing methods. Samples from the

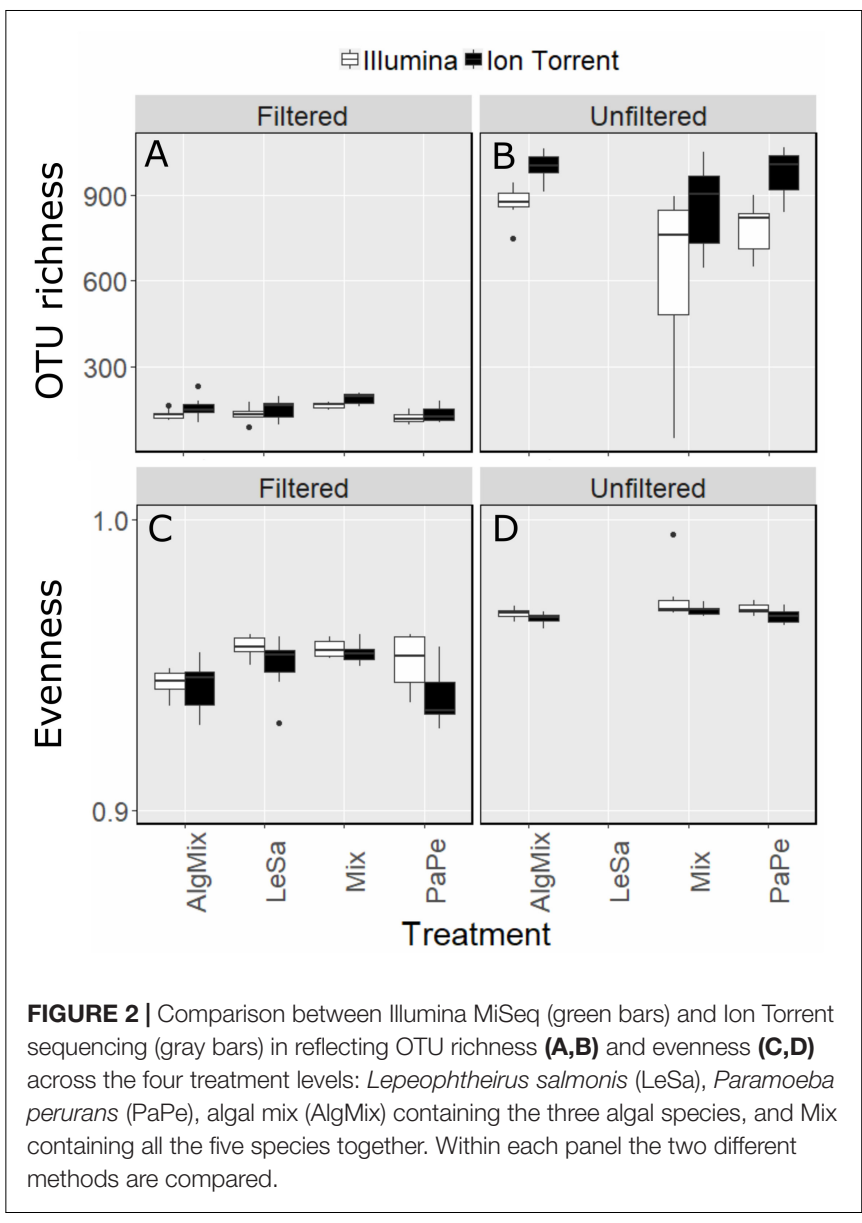

L. salmonis incubation in unfiltered samples failed sequencing on both platforms, presumably due to problems with the first round $\mathrm{PCR}$.

The OTUs of our algal species of interest were the most abundant reads amplified from the filtered samples containing the algal mix, and their identity was validated by a nucleotide BLAST search against the Genbank database on the NCBI website. As expected, both sequencing methods resulted in OTUs that could be assigned taxonomic identities to our target algae species with equal efficiency. However, some algal assignments were different to the known identity of the target organisms we introduced. The OTU that was assigned to Prymnesium was first mis-assigned as $P$. nemathicium using SILVA but later correctly identified as Prymnesium parvum by the top NCBI BLAST hit (100\% sequence identity). As such this OTU was identified as $P$. parvum for downstream analysis. The validation of the Pseudo-nitzschia spp. identities was more ambiguous since our target species, Pseudo-nitzschia seriata and P. delicatissima, were assigned as Pseudo-nitzschia australis and cuspidata using SILVA and these taxonomic identities were confirmed by the top BLAST hits for these OTUs. L. salmonis and P. perurans were correctly assigned to species level with reference to both SILVA and NCBI BLAST.

Once the OTU tables from each method were collapsed to avoid multiple identical taxonomic assignments, the Ion Torrent 


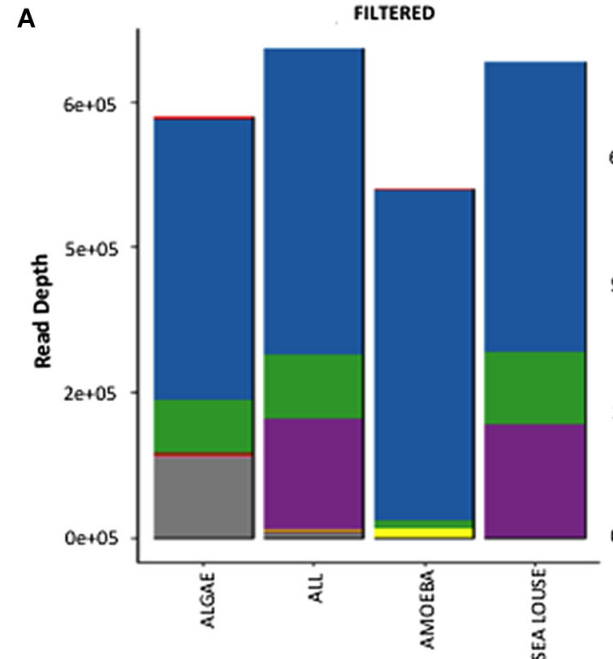

B

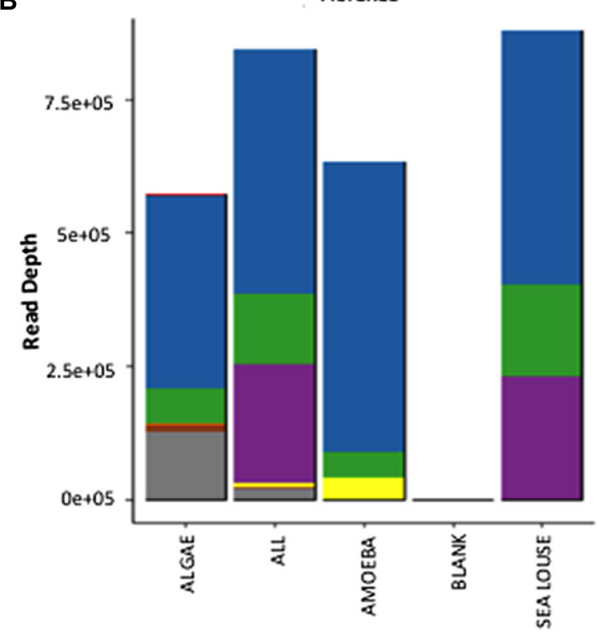

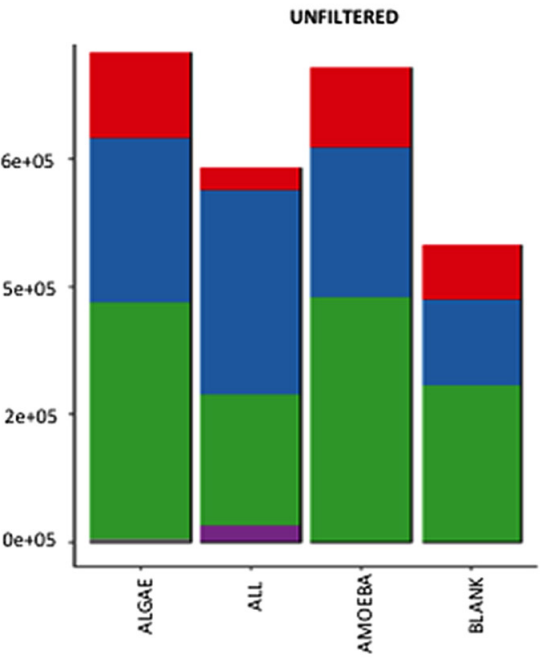

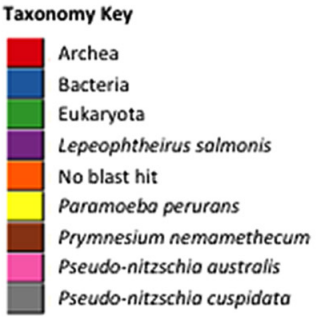

FIGURE 3 | Absolute read numbers for samples grouped according to treatment and split for medium type. Color code identifies different taxonomic groups. (A) Represents the MiSeq data, (B) the data from the lon Torrent run.

data appeared to capture slightly more diversity across all samples (2463 OTUs) compared to MiSeq (2277 OTUs). This was also observed on a per treatment basis (Figures 2A,B) as the effect of method was found significant (GLM, F-ratio $=13.5, p<0.001)$ having accounted for medium type (pre-filtered versus unfiltered seawater). As expected the number of OTUs was much larger in the unfiltered medium type than the pre-filtered samples (GLM, F-ratio $=1329.8, p<0.001)$. The effect of method depended on the medium type with the Illumina MiSeq performing significantly better than Ion Torrent in detecting more OTUs in the unfiltered sea water medium compared to the filtered one (GLM, F-ratio $=16.5, p<0.001)$. Although sample evenness (i.e., how evenly reads are distributed across OTUs) was significantly lower across treatments (Figures 2C,D) for the Ion Torrent method $($ GLM, F-ratio $=17.2, p<0.001)$ for both filtered and unfiltered medium (GLM, F-ratio $=1.3, p=0.249$ ), nevertheless the effect size was very small as in both methods evenness ranged between 0.93 and 0.98 (evenness can range from 0 to 1 ). Complete
OTU tables can be found for each of the sequencing methods in the Supplementary Material.

\section{Sensitivity of Illumina MiSeq Versus Ion Torrent to Reflect Sample Composition}

Both methods detected similar proportions of taxonomic groups considering the absolute read numbers across incubation treatments (Figure 3). Treatments containing unfiltered medium were more diverse in other taxonomic groups including bacteria, archaea and eukaryotes other than our target species. By comparison, in the filtered samples only bacteria were present apart from our target species. The Illumina Miseq and Ion Torrent methods were identical in depicting sample OTU composition as seen both by the relative contribution of taxa they detect (Figure 3) as well as the pairwise similarity between treatments (Supplementary Figure S1). Specifically, community composition in the unfiltered samples was clearly separated from 


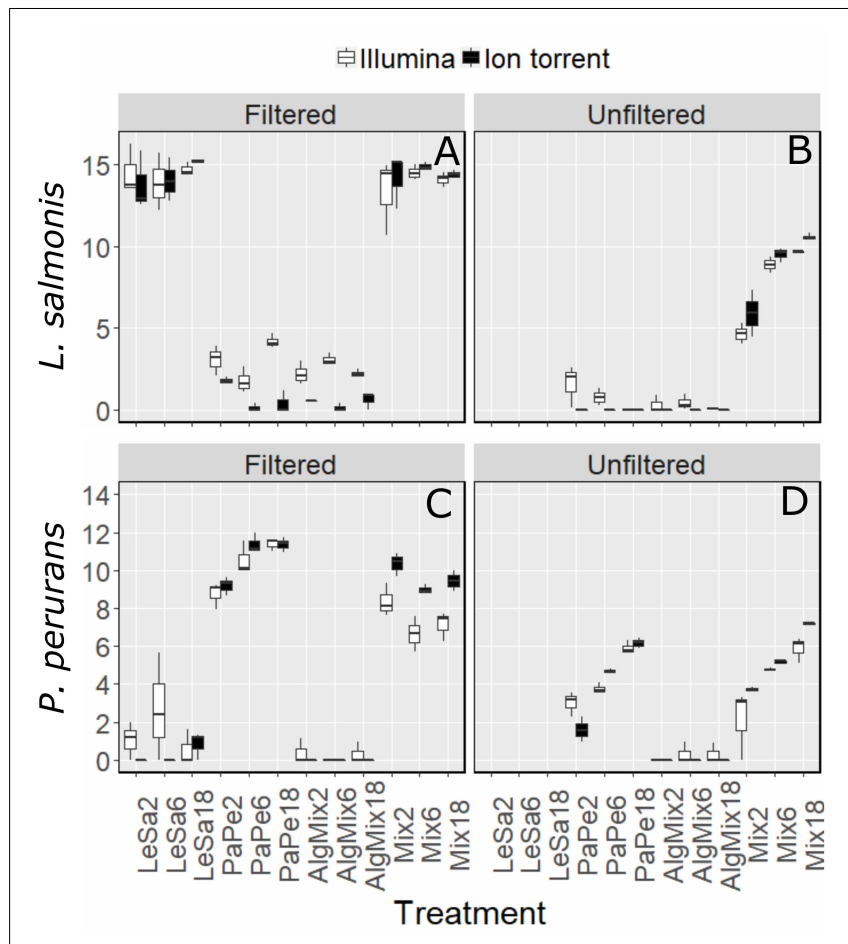

FIGURE 4 | Variability in the read numbers of the two target parasite species namely Paramoeba perurans (A,B) and Lepeophtheirus salmonis (C,D) across the different treatments (see Figure $\mathbf{1}$ for treatment abbreviations). Within each treatment, the reads of each species were compared between the two sequencing methods Illumina (green bars) and lon Torrent (gray bars) and between filtered and unfiltered marine plankton samples. For the unfiltered (not pre-filtered) water, increasing relative abundances of reads for target organisms are evident as their concentration is increased in the original sample. A similar pattern is not observed for results from prefiltered water.

Low levels of cross contamination between the two target species

( $P$. perurans reads in $L$. salmonis samples and vice versa) are also observed.

the filtered samples using data from either sequencing method (Supplementary Figure S1). Also, samples from different treatments were grouped together at equal similarity within each method, showing more pronounced separation of treatments in the pre-filtered medium that the unfiltered one. The greater similarity observed in the unfiltered treatments was because of the interference of the non-target organisms in the seawater sample that were common to all unfiltered samples.

\section{Sensitivity of MiSeq Versus Ion Torrent to Reflect Differences in Relative Abundances of Target Organisms Between Treatments}

In the pre-filtered samples, the two sequencing methods were not able to reflect the different abundance levels of the two parasite species L. salmonis and $P$. perurans (Figures $4 \mathrm{~A}, \mathrm{C}$ and Table 1) and the same was true for the harmful microalgae species (Figures 5A,C,D and Table 1). In the unfiltered samples, however, the two methods were able to detect the tripling in abundance between the three abundance treatments $(2,6$, and
TABLE 1 | The F-ratio and corresponding confidence level (* for 95\%, ** for 99\%, and $* * *$ for $99.9 \%$ level) shows whether there is a significant difference between the 3 abundance levels for a given species within the treatment it was present.

\begin{tabular}{|c|c|c|c|c|}
\hline \multirow[b]{2}{*}{ Medium } & \multirow[b]{2}{*}{ Species } & \multirow[b]{2}{*}{ Treatment } & \multicolumn{2}{|c|}{ F-ratio and significance level } \\
\hline & & & Illumina MiSeq & Ion Torrent \\
\hline \multirow[t]{10}{*}{ Filtered } & L. salmonis & LeSa & 0.275 & 1.047 \\
\hline & & Mix & 0.537 & 0.368 \\
\hline & P. perurans & $\mathrm{PaPe}$ & $11.77^{* *}$ & $22.51^{* *}$ \\
\hline & & Mix & 3.228 & $5.562 *$ \\
\hline & P. australis & AlgMix & 0.420 & 0.584 \\
\hline & & Mix & 2.825 & 0.226 \\
\hline & P. cuspidata & AlgMix & 1.348 & 0.2861 \\
\hline & & Mix & 4.998 & $6.910^{*}$ \\
\hline & P. parvum & AlgMix & 1.354 & 2.119 \\
\hline & & Mix & $5.341^{*}$ & 0.5548 \\
\hline \multirow[t]{10}{*}{ Unfiltered } & L. salmonis & LeSa & N/A & $\mathrm{N} / \mathrm{A}$ \\
\hline & & Mix & $102.67^{* * *}$ & $14.818^{* *}$ \\
\hline & P. perurans & $\mathrm{PePa}$ & $34.468^{* * *}$ & $54.006^{* *}$ \\
\hline & & Mix & $8.838^{*}$ & $526.000^{* * *}$ \\
\hline & P. australis & AlgMix & 2.924 & 5.799* \\
\hline & & Mix & $19.838^{* *}$ & 1.178 \\
\hline & P. cuspidata & AlgMix & $26.257^{* *}$ & $18.446^{* *}$ \\
\hline & & Mix & 0.997 & 4.137 \\
\hline & P. parvum & AlgMix & $17.783^{* *}$ & $24.546^{* *}$ \\
\hline & & Mix & 0.866 & $16.673^{* *}$ \\
\hline
\end{tabular}

Dark boxes indicate significant differences and also correct directionality of the effect meaning that an increase in the treatment abundance level corresponded to an increase in the reads that were sequenced.

18) for all target species apart from $P$. australis (Figures 4B,D, 5B,D,F and Table 1). Specifically, Ion Torrent was successful in 6 out of the seven sequenced treatments whereas Illumina MiSeq for 5 out of seven treatments (see Table 1). We did observe some cross contamination between samples (Figures 4, 5) - a feature more prominent on the Illumina platform than the Ion Torrrent platform.

\section{DISCUSSION}

The data we present suggest that eDNA metabarcoding of the 18S SSU rDNA v9 region can sensitively and specifically identify multiple aquaculture pathogens from the complex mixture of organisms present in seawater. Furthermore, our benchmarking indicates that such detection can be achieved using a low-cost, scalable Ion Torrent sequencing platform - in some cases with better results than the Illumina gold standard. Our findings suggest that eDNA metabarcoding may represent a valuable tool in the hands of producers and regulators alike.

As our data suggest, however, several challenges remain. Taxonomic ambiguity around the assignment of the target HAB species (P. parvum, Pseudo-nitchzia sp.) highlights the difficulty of using short 18S rDNA amplicons to assign taxa to species level. Although the $18 \mathrm{~S}$ V9 SSU region is thought to be useful for detecting global protist diversity (Amaral-Zettler et al., 2009), one size rarely fits all when choosing metabarcoding markers. 


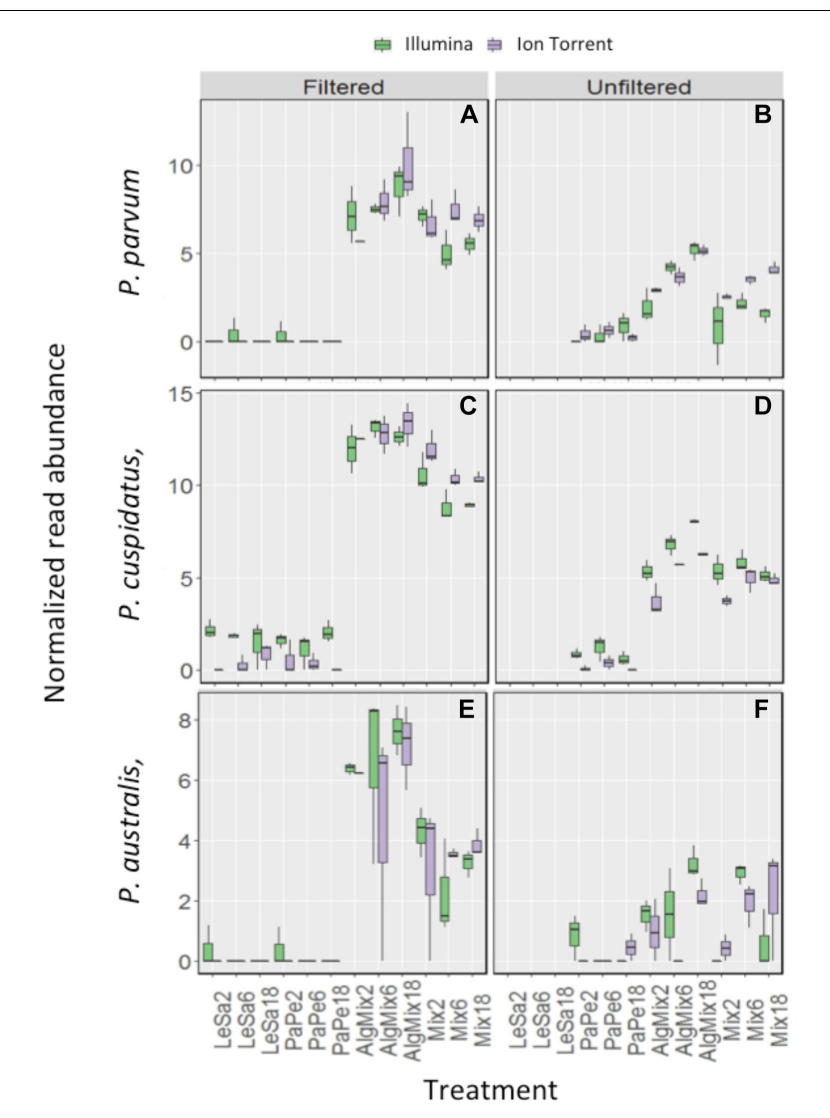

FIGURE 5 | Variability in the read numbers of the three target microalgal species namely the two Pseudo-nitzschia species that were assigned as $P$. cuspidata and $P$. australis, but which were in fact $P$. delicatissima and P. seriata (A-D) and Prymnesium parvum (E,F) across the different treatments (see Figure $\mathbf{1}$ for treatment abbreviations). Within each treatment, the reads of each species were compared between the two sequencing methods Illumina MiSeq and lon Torrent and between filtered and unfiltered marine plankton samples. For the non-prefiltered seawater samples, increasing relative abundances of reads for the target organisms is evident as their concentration is increased in the original sample for every organism except $P$. australis. A similar pattern is not observed in prefiltered seawater samples. Low levels of cross contamination between the target species ( $P$. perurans an $L$. salmonis reads in algal samples) is also observed.

The 18S SSU rDNA V4 region has also been shown to have high global eukaryotic discriminatory power (Hugerth et al., 2014). However, to an extent markers must be targeted at particular groups, and the $23 \mathrm{~S}$ rDNA locus is thought to provide better discrimination for algae in particular (Yoon et al., 2016). Nonetheless the $18 \mathrm{~S}$ v9 region we tested did provide excellent discrimination for $L$. salmonis and $P$. perurans which together account for persistent morbidity in salmonid aquaculture (Young et al., 2008; Torrissen et al., 2013; Ellis et al., 2016). In future work, it may be more appropriate to target specific organisms with tailored molecular probes, or to deploy amplicon-seq using longer read sequencing technologies to improve global species resolution. However, the former precludes the identification of novel pathogenic agents, while the latter remains too expensive to be widely adopted (Franzen et al., 2015). In addition, there is now substantial interest in adopting approaches that can be deployed "in situ" to allow point-of-care diagnosis. So-called "lab in a suitcase" approaches, facilitated by miniaturized and portable PCR, qPCR and sequencing (Petralia and Conoci, 2017), have made a substantial recent impact in recent biomedical contexts, such as tracking viral outbreaks (Quick et al., 2016). For salmonid aquaculture end-users, often located at remote sites far from laboratory facilities, rapid decisions are key to mitigate losses from disease outbreaks caused by agents like $P$. perurans, where early treatment is vital (Downes et al., 2015).

Although the absolute quantification of abundances in community samples remains a challenge is molecular ecology, the normalization of read data increases their accurateness in representing actual species abundances, as we have done here, so that experimentally induced differences can be more reliably reflected (Weber and Pawlowski, 2013). Nonetheless, PCR artifacts relating to runaway amplification of relatively abundant target species templates in pre-filtered water may have negatively impacted relative quantification in our study. However, in more biologically realistic samples (normal, unfiltered seawater) we were able to detect the expected increase in relative abundances in each sample according to our dilution series. Metabarcoding is poorly suited to achieving absolute abundance estimates as a direct link between the numbers of amplicons sequenced and the quantity of template in the original sample is difficult to establish - especially because two rounds of PCR are required before sequencing (Bista et al., 2018). In such cases, a single round, taxon targeted qPCR might be more appropriate (e.g., Berger and Aubin-Horth, 2018). However, PCR always carries an intrinsic risk (e.g., via differential presence of PCR inhibitors in different samples) in quantification from real-world samples (Murray et al., 2015). PCR-free approaches, metagenomic or probe-based for example, may be more appropriate for the detection of absolute biomass abundance (Bista et al., 2018).

Our benchmarking of Ion Torrent and Illumina platforms are in line with the reports of others who have done so for ampliconseq datasets, especially in relation to higher error rates in the former (Salipante et al., 2014; D’Amore et al., 2016). However, both platforms were able to recover community compositions in our data with similar accuracy, as well as recover relative quantities of target species (D'Amore et al., 2016). The ability to run a single low output Ion-Torrent chip at a fraction of the price of the Illumina MiSeq to rapidly process only 20-40 samples, means that this technology may be more easy to adopt in a diagnostic context. Several newer Illumina models (MiniSeq, ISeq) are coming to market to address the issue of scalability. Cost may not be the only consideration, however, we were able to detect a higher rate of apparent contamination between samples from data generated using the Illumina platform (e.g., Figures 4, 5). One possible source of such contamination in our experiment is bleeding between sequence clusters, a known feature of the Illumina platform (e.g., Schnell et al., 2015), a further potential disadvantage of the Illumina sequencing approach using the exisiting MiSeq chemistry.

Our data, and that of others, does suggest that eDNA metabarcoding may soon become a useful tool for monitoring biological threats to aquaculture. Next steps could include field 
trials of such methodologies and corroboration with direct count data to fully validate the approach for use in the industry.

\section{AUTHOR CONTRIBUTIONS}

ML, SS, and KP conceived the study. LP, MD, AK, IR, and TD undertook the research and analyses. $\varnothing \mathrm{K}-\mathrm{H}$ supplied materials and reagents. LP, SS, KP, and ML wrote the paper.

\section{FUNDING}

ML and SS are grateful for financial support from a Wellcome Trust ISSF Catalyst project (105614/Z/14/Z). ML was also grateful for financial support from BBSRC project from grants BB/N024028/1 and BB/P001203/1 that also contributed to the project. KP was grateful for a strategic grant from the Faculty of

\section{REFERENCES}

Amaral-Zettler, L. A., McCliment, E. A., Ducklow, H. W., and Huse, S. M. (2009). A method for studying protistan diversity using massively parallel sequencing of V9 hypervariable regions of small-subunit ribosomal RNA Genes. PLoS One 4:9. doi: 10.1371/journal.pone.0006372

Berger, C. S., and Aubin-Horth, N. (2018). An eDNA-qPCR assay to detect the presence of the parasite Schistocephalus solidus inside its three spine stickleback host. J. Exp. Biol. 221:jeb178137. doi: 10.1242/jeb. 178137

Bista, I., Carvalho, G. R., Tang, M., Walsh, K., Zhou, X., Hajibabaei, M., et al. (2018). Performance of amplicon and shotgun sequencing for accurate biomass estimation in invertebrate community samples. Mol. Ecol. Resour. doi: 10.1111/ 1755-0998.12888 [Epub ahead of print].

Bohmann, K., Evans, A., Gilbert, M. T., Carvalho, G. R., Creer, S., Knapp, M., et al. (2014). Environmental DNA for wildlife biology and biodiversity monitoring. Trends Ecol. Evol. 29, 358-367. doi: 10.1016/j.tree.2014.04.003

Caporaso, J. G., Kuczynski, J., Stombaugh, J., Bittinger, K., Bushman, F. D., Costello, E. K., et al. (2010). QIIME allows analysis of high-throughput community sequencing data. Nat. Methods 7, 335-336. doi: 10.1038/nmeth. f.303

Clarke, K. R., and Gorley, R. N. (2006). PRIMER v6: User Manual/Tutorial. Plymouth: PRIMER-E, 192.

Clooney, A. G., Fouhy, F., Sleator, R. D., O', Driscoll A, Stanton, C., Cotter, P. D., et al. (2016). Comparing apples and oranges?: next generation sequencing and its impact on microbiome analysis. PLoS One 11:e0148028. doi: 10.1371/journal. pone. 0148028

D’Amore, R., Ijaz, U. Z., Schirmer, M., Kenny, J. G., Gregory, R., Darby, A. C., et al. (2016). A comprehensive benchmarking study of protocols and sequencing platforms for 16S rRNA community profiling. BMC Genomics 17:20. doi: 10 . 1186/s12864-015-2194-9

Downes, J. K., Henshilwood, K., Collins, E. M., Ryan, A., Connor, I. O., Rodger, H. D., et al. (2015). A longitudinal study of amoebic gill disease on a marine Atlantic salmon farm utilising a real-time PCR assay for the detection of Neoparamoeba perurans. Aquacult. Environ. Interact. 7, 239-251. doi: 10.3354/ aei00150

Edgar, R. C. (2010). Search and clustering orders of magnitude faster than BLAST. Bioinformatics 26, 2460-2461. doi: 10.1093/bioinformatics/ btq461

Eiler, A., Drakare, S., Bertilsson, S., Pernthaler, J., Peura, S., Rofner, C., et al. (2013). Unveiling distribution patterns of freshwater phytoplankton by a next generation sequencing based approach. PLoS One 8:10. doi: 10.1371/journal. pone.0053516

Ellis, T., Turnbull, J. F., Knowles, T. G., Lines, J. A., and Auchterlonie, N. A. (2016). Trends during development of scottish salmon farming: an example of
Biosciences, Fisheries, and Economics, University of Tromso to support the experimental testing and Ion PGM sequencing.

\section{ACKNOWLEDGMENTS}

We thanks to Catherine Collins of Marine Scotland Science for providing the $P$. perurans and to Nina Lundholm of Natural History Museum of Denmark for providing P. seriata used in this study.

\section{SUPPLEMENTARY MATERIAL}

The Supplementary Material for this article can be found online at: https://www.frontiersin.org/articles/10.3389/fmicb. 2018.03009/full\#supplementary-material

sustainable intensification? Aquaculture 458, 82-99. doi: 10.1016/j.aquaculture. 2016.02.012

Ficetola, G. F., Miaud, C., Pompanon, F., and Taberlet, P. (2008). Species detection using environmental DNA from water samples. Biol. Lett. 4:423. doi: 10.1098/ rsbl.2008.0118

Franzen, O., Hu, J. Z., Bao, X. L., Itzkowitz, S. H., Peter, I., and Bashir, A. (2015). Improved OTU-picking using long-read 16S rRNA gene amplicon sequencing and generic hierarchical clustering. Microbiome 3:14.

Gomes, G. B., Hutson, K. S., Domingos, J. A., Chung, C., Hayward, S., Miller, T. L., et al. (2017). Use of environmental DNA (eDNA) and water quality data to predict protozoan parasites outbreaks in fish farms. Aquaculture 479, 467-473. doi: 10.1016/j.aquaculture.2017.06.021

Hadziavdic, K., Lekang, K., Lanzen, A., Jonassen, I., Thompson, E. M., and Troedsson, C. (2014). Characterization of the 18S rRNA gene for designing universal eukaryote specific primers. PLoS One 9:e87624. doi: 10.1371/journal. pone.0087624

Hinder, S. L., Hays, G. C., Brooks, C. J., Davies, A. P., Edwards, M., Walne, A. W., et al. (2011). Toxic marine microalgae and shellfish poisoning in the British isles: history, review of epidemiology, and future implications. Environ. Health 10:12. doi: 10.1186/1476-069X-10-54

Hugerth, L. W., Muller, E. E., Hu, Y. O., Lebrun, L. A., Roume, H., Lundin, D., et al. (2014). Systematic design of $18 \mathrm{~S}$ rRNA gene primers for determining eukaryotic diversity in microbial consortia. PLoS One 9:11. doi: 10.1371/journal. pone.0095567

Joshi, N. A., and Fass, J. N. (2011). Sickle: A Sliding-Window, Adaptive, QualityBased Trimming tool for FastQ Files. Available at https://github.com/najoshi/ sickle

Lahens, N. F., Ricciotti, E., Smirnova, O., Toorens, E., Kim, E. J., Baruzzo, G., et al. (2017). A comparison of Illumina and Ion Torrent sequencing platforms in the context of differential gene expression. BMC Genomics 18:602. doi: 10.1186/ s12864-017-4011-0

Langmead, B., and Salzberg, S. L. (2012). Fast gapped-read alignment with Bowtie 2. Nat. Methods 9, 357-U354. doi: 10.1038/nmeth.1923

Liu, Y. J., and Bjelland, H. V. (2014). Estimating costs of sea lice control strategy in Norway. Prev. Vet. Med. 117, 469-477. doi: 10.1016/j.prevetmed.2014.08.018

Love, M. I., Huber, W., and Anders, S. (2014). Moderated estimation of fold change and dispersion for RNA-seq data with DESeq2. Genome Biol. 15:38. doi: 10.1186/s13059-014-0550-8

Mahon, A. R., Jerde, C. L., Galaska, M., Bergner, J. L., Chadderton, W. L., Lodge, D. M., et al. (2013). Validation of eDNA Surveillance Sensitivity for Detection of Asian Carps in Controlled and Field Experiments. PLoS One 8:6. doi: 10.1371/ journal.pone.0058316

Manning, S. R., and La Claire, J. W. (2010). Prymnesins: toxic metabolites of the golden alga, Prymnesium parvum carter (Haptophyta). Mar. Drugs 8, 678-704. doi: $10.3390 / \mathrm{md} 8030678$ 
McMurdie, P. J., and Holmes, S. (2013). phyloseq: an r package for reproducible interactive analysis and graphics of microbiome census data. PLoS One 8:11. doi: 10.1371/journal.pone.0061217

McMurdie, P. J., and Holmes, S. (2014). Waste not, want not: why rarefying microbiome data is inadmissible. Plos Comput. Biol. 10:12. doi: 10.1371/journal. pcbi. 1003531

Murray, D. C., Coghlan, M. L., and Bunce, M. (2015). From benchtop to desktop: important considerations when designing amplicon sequencing workflows. PLoS One 10:21. doi: 10.1371/journal.pone.0124671

Nathan, L. M., Simmons, M., Wegleitner, B. J., Jerde, C. L., and Mahon, A. R. (2014). Quantifying environmental DNA signals for aquatic invasive species across multiple detection platforms. Environ. Sci. Technol. 48, 12800-12806. doi: 10.1021/es5034052

Olson, Z. H., Briggler, J. T., and Williams, R. N. (2012). An eDNA approach to detect eastern hellbenders (Cryptobranchus a. Alleganiensis) using samples of water. Wildlife Res. 39, 629-636. doi: 10.1071/WR12114

Petralia, S., and Conoci, S. (2017). PCR technologies for point of care testing: progress and perspectives. ACS Sens. 2, 876-891. doi: 10.1021/acssensors. 7 b00299

Quast, C., Pruesse, E., Yilmaz, P., Gerken, J., Schweer, T., Yarza, P., et al. (2013). The SILVA ribosomal RNA gene database project: improved data processing and web-based tools. Nucleic Acids Res. 41, D590-D596. doi: 10.1093/nar/gks1219

Quick, J., Loman, N. J., Duraffour, S., Simpson, J. T., Severi, E., Cowley, L., et al. (2016). Real-time, portable genome sequencing for Ebola surveillance. Nature 530, 228. doi: 10.1038/nature16996

R Core Team (2016). R: A Language and Environment for Statistical Computing. Vienna: R Foundation for Statistical Computing.

Salipante, S. J., Kawashima, T., Rosenthal, C., Hoogestraat, D. R., Cummings, L. A., Sengupta, D. J., et al. (2014). Performance comparison of illumina and ion torrent next-generation sequencing platforms for $16 \mathrm{~S}$ rRNA-based bacterial community profiling. Appl. Environ. Microbiol. 80, 7583-7591. doi: 10.1128/ AEM.02206-14

Sassoubre, L. M., Yamahara, K. M., Gardner, L. D., Block, B. A., and Boehm, A. B. (2016). Quantification of environmental DNA (eDNA) shedding and decay rates for three marine fish. Environ. Sci. Technol. 50, 10456-10464. doi: 10.1021/acs.est.6b03114

Schmieder, R., and Edwards, R. (2011). Quality control and preprocessing of metagenomic datasets. Bioinformatics 27, 863-864. doi: 10.1093/ bioinformatics/btr026

Schnell, I. B., Bohmann, K., and Gilbert, M. T. P. (2015). Tag jumps illuminated reducing sequence-to-sample misidentifications in metabarcoding studies. Mol. Ecol. Resour. 15, 1289-1303. doi: 10.1111/1755-0998.12402

Smayda, T. J. (2006). Harmful Algal Bloom Communities in Scottish Coastal Waters: Relationship to Fish Farming and Regional Comparisons - A Review: Paper 2006/3. Edinburgh: Scottish Government.
Taberlet, P., Coissac, E., Hajibabaei, M., and Rieseberg, L. H. (2012). Environmental DNA. Mol. Ecol. 21, 1789-1793. doi: 10.1111/j.1365-294X.2012.05542.x

Thomsen, P. F., Kielgast, J., Iversen, L. L., Wiuf, C., Rasmussen, M., Gilbert, M. T., et al. (2012). Monitoring endangered freshwater biodiversity using environmental DNA. Mol. Ecol. 21, 2565-2573. doi: 10.1111/j.1365-294X.2011. 05418.x

Torrissen, O., Jones, S., Asche, F., Guttormsen, A., Skilbrei, O. T., Nilsen, F., et al. (2013). Salmon lice - impact on wild salmonids and salmon aquaculture. J. Fish Dis. 36, 171-194. doi: 10.1111/jfd.12061

Weber, A. A. T., and Pawlowski, J. (2013). Can abundance of protists be inferred from sequence data: a case study of foraminifera. PLoS One 8:e56739. doi: 10.1371/journal.pone.0056739

Wood, S. A., Smith, K. F., Banks, J. C., Tremblay, L. A., Rhodes, L., Mountfort, D., et al. (2013). Molecular genetic tools for environmental monitoring of New Zealand's aquatic habitats, past, present and the future. New Zeal. J. Mar. Freshw. Res. 47, 90-119. doi: 10.1080/00288330.2012.745885

Yoon, T. H., Kang, H. E., Kang, C. K., Lee, S. H., Ahn, D. H., Park, H., et al. (2016). Development of a cost-effective metabarcoding strategy for analysis of the marine phytoplankton community. Peerj 4:20. doi: 10.7717/peerj. 2115

Young, N. D., Dykova, I., Nowak, B. F., and Morrison, R. N. (2008). Development of a diagnostic PCR to detect Neoparamoeba perurans, agent of amoebic gill disease. J. Fish Dis. 31, 285-295. doi: 10.1111/j.1365-2761.2008.00903.x

Yu, L. Y., Zhang, W. J., Liu, L. M., and Yang, J. (2015). Determining microeukaryotic plankton community around xiamen island, southeast China, using illumina miseq and PCR-DGGE techniques. PLoS One 10:16. doi: 10. 1371/journal.pone.0127721

Zamor, R. M., Glenn, K. L., and Hambright, K. D. (2012). Incorporating molecular tools into routine $\mathrm{HAB}$ monitoring programs: using $\mathrm{qPCR}$ to track invasive Prymnesium. Harmful Algae 15, 1-7. doi: 10.1016/j.hal.2011.10.028

Zerbino, D. R., and Birney, E. (2008). Velvet: algorithms for de novo short read assembly using de Bruijn graphs. Genome Res. 18, 821-829. doi: 10.1101/gr. 074492.107

Conflict of Interest Statement: The authors declare that the research was conducted in the absence of any commercial or financial relationships that could be construed as a potential conflict of interest.

Copyright (c) 2018 Peters, Spatharis, Dario, Dwyer, Roca, Kintner, Kanstad-Hanssen, Llewellyn and Praebel. This is an open-access article distributed under the terms of the Creative Commons Attribution License (CC BY). The use, distribution or reproduction in other forums is permitted, provided the original author(s) and the copyright owner(s) are credited and that the original publication in this journal is cited, in accordance with accepted academic practice. No use, distribution or reproduction is permitted which does not comply with these terms. 